CLINICAL STUDY

\title{
Lower heart rate variability is associated with higher plasma concentrations of IL-6 in type 1 diabetes
}

\author{
J-M González-Clemente, C Vilardell, M Broch ${ }^{1}$, A Megia ${ }^{1}$, A Caixàs, O Giménez-Palop, C Richart ${ }^{1}$, I Simón ${ }^{1}$, \\ A Martínez-Riquelme ${ }^{2}$, J Arroyo, D Mauricio and J Vendrell ${ }^{1}$ \\ Department of Diabetes, Endocrinology and Nutrition, Hospital de Sabadell, Parc Taulí s/n, 08208 Sabadell, Spain, ${ }^{1}$ Diabetes and Research Unit, Hospital \\ Universitari de Tarragona Joan XXIII, Universitat Rovira i Virgili, 43007 Tarragona, Spain and ${ }^{2}$ Associació Catalana de Diabetis, O8017 Barcelona, Spain \\ (Correspondence should be addressed to J-M González-Clemente; Email: jmgonzalez@cspt.es)
}

\begin{abstract}
Objective: In type 1 diabetes, cardiovascular autonomic neuropathy (CAN) is associated with cardiovascular risk factors related to insulin resistance, which in turn are associated with low-grade systemic inflammation. Reduced heart rate variability (HRV) is considered one of the first indicators of CAN. Since the autonomic nervous system interacts with systemic inflammation, we evaluated CAN to study its possible association with low-grade systemic inflammation.

Design: Cross-sectional study of a group of 120 subjects diagnosed with type 1 diabetes mellitus 14 years before.

Methods: Information recorded: 1) clinical characteristics: sex, age, body mass index, waist-to-hip ratio (WHR), blood pressure (BP), smoking, alcohol intake, insulin dose, HbA1c, and lipid profile; 2) plasma levels of soluble fractions of tumour necrosis factor $\alpha$ receptors 1 and 2, IL-6, and C-reactive protein; 3) insulin resistance by estimation of the glucose disposal rate (eGDR); and 4) tests for CAN: HRV in response to deep breathing (E/I ratio), HRV in response to the Valsalva maneuver, and changes in systolic BP responding to standing.

Results: A significant negative correlation was found between $E / I$ ratio and plasma concentrations of IL-6 $(r=-0.244, P=0.032)$, which remained significant after adjusting for potential confounding factors (age, sex, HbA1c, WHR, diastolic BP, triglycerides, HDL-cholesterol, retinopathy, nephropathy, peripheral neuropathy, insulin dose, and smoking; $r=-0.231, P=0.039$ ). No other significant associations were found between inflammation-related proteins, tests for CAN, and eGDR.

Conclusions: These findings suggest a link between low-grade inflammation and early alterations of CAN in type 1 diabetes and may be of importance in the pathogenesis of CAN and/or its clinical implications.
\end{abstract}

European Journal of Endocrinology 157 31-38

\section{Introduction}

In subjects with diabetes mellitus, damage to both parasympathetic and sympathetic fibers innervating the cardiovascular system produces the cardiovascular autonomic neuropathy (CAN). CAN clearly entails an increase in mortality and an acceleration of other microvascular complications (1). In CAN, the disruption of the parasympathetic nervous system is usually detected earlier than that of the sympathetic nervous system, and reduced heart rate variability (HRV) is noticed as its first indicator (1). To assess CAN, the combination of the following three tests, with wellestablished reproducibility, reliability, and normal values, were proposed in 1992 (2), subsequently supported (3), and then used in the Diabetes Control and Complications Trial (4): 1) HRV in response to deep breathing, 2) HRV in response to the Valsalva maneuver, and 3) systolic blood pressure (sBP) responding to standing. The first test is mainly considered to reflect parasympathetic activity, the second is influenced by both parasympathetic and sympathetic activity, and the third is used to assess sympathetic activity (1).

In type 1 diabetes, CAN is associated with some cardiovascular risk factors included in the metabolic syndrome such as higher BP or fasting triglycerides or lower HDL-cholesterol $(5,6)$, supporting a role of nerve ischemia in its pathogenesis. This association is not surprising, since these cardiovascular risk factors are associated with insulin resistance and, furthermore, insulin resistance in type 1 diabetes has also been related to the presence of other microvascular complications (7-9) and coronary heart disease $(8,10)$. In addition, in type 1 diabetes, these cardiovascular risk factors have recently been associated with a low-grade systemic inflammation response (11). This finding is in accordance with accumulated evidence suggesting that insulin resistance is associated with an increase in the plasma concentrations of several proteins, such as 
tumor necrosis factor (TNF)- $\alpha$ (or the soluble fractions of its receptors type 1 and 2, sTNFR1 and sTNFR2 respectively), IL-6, and C-reactive protein (CRP; (12)). Whether this low-grade systemic inflammation leads to insulin resistance or whether insulin resistance brings about a condition of low-grade systemic inflammation is still unclear. However, it has been speculated that this low-grade systemic inflammation could be implicated not only in the pathogenesis of insulin resistance and the metabolic syndrome (12), but also in the pathogenesis of diabetic microvascular complications $(8,13)$ and cardiovascular disease $(8,10)$ in type 1 diabetes.

Several studies have shown that a sympathovagal imbalance (manifested by depression of the parasympathetic nervous activity) associates with insulin resistance $(14,15)$, some cardiovascular risk factors included in the metabolic syndrome $(5,16,17)$, and the low-grade systemic inflammation accompanying insulin resistance $(18,19)$. Although CAN is a well-known complication in type 1 diabetes, to the best of our knowledge, no previous study has addressed whether indexes of CAN are associated with low-grade systemic inflammation, taking into account that insulin resistance may also be present in type 1 diabetes (20). Our main hypothesis was that in type 1 diabetes, early signs of CAN, usually detected as a parasympathetic dysfunction (i.e. a reduced HRV), would be associated with insulin resistance and the abovementioned inflammation-related plasma proteins. For testing this hypothesis, we carried out a cross-sectional study in a group of subjects with type 1 diabetes after 14 years of evolution of their disease.

\section{Subjects and methods}

A group of subjects with type 1 diabetes, all having been diagnosed 14 years before following a previous protocol (21), were consecutively recruited in 15 hospital centers in Catalonia, northeastern Spain. They were previously studied with respect to the likely role of inflammation on diabetic neuropathy (9). None of them had any conditions that increased inflammation-related plasma proteins (e.g., acute or chronic inflammatory or infectious diseases) or had received anti-platelet, antiinflammatory, or lipid-lowering treatment. None of them had any clinically proven cardiovascular diseases, cardiac arrhythmia, or were on drugs affecting the autonomic nervous system.

The study protocol was approved by the local ethics committee in each hospital and was conducted according to the principles of the Declaration of Helsinki. All subjects gave their informed consent before participating in the study. After an overnight fast, venous blood samples were taken and aliquots of plasma and serum were stored at $-70{ }^{\circ} \mathrm{C}$ until processing. The following information was recorded using a predefined standardized form: sex, age, diabetes duration after puberty, body mass index (BMI), waist-to-hip ratio
(WHR), BP, cigarette smoking, alcohol intake, insulin dose, use of any other medical treatment, HbAlc, lipid profile, plasma concentrations of sTNFR1, sTNFR2, IL-6, high-sensitivity CRP, and microvascular complications. To estimate insulin resistance, we used the formula proposed by Williams et al. (20) validated in subjects with type 1 diabetes. This formula yielded an estimation of the glucose disposal rate (eGDR), taking into account glycemic control, WHR, and BP.

All laboratory determinations were performed at a central facility. HbA1c was determined in capillary blood by immunoassay (DCA 2000, Bayer AG). Total serum cholesterol, triglycerides, and HDL-cholesterol were measured using standard enzymatic methods. LDLcholesterol was calculated using the Friedewald formula. Plasma sTNFR 1 and sTNFR2 were determined by a solidphase enzyme-amplified sensitivity immunoassay (Medgenix sTNFR1-EASIA, sTNFR2-EASIA, BioSource Europe, Fleurus, Belgium). The lower limit of detection was $50 \mathrm{pg} / \mathrm{ml}$ for sTNFR 1 and $0.1 \mathrm{ng} / \mathrm{ml}$ for sTNFR2, and the intra- and interassay coefficients of variation were $<7$ and $<9 \%$ respectively. TNF- $\alpha$ does not interfere with these assays and sTNFR1 assay does not cross-react with sTNFR2. IL -6 was determined by an ultrasensitive solidphase enzyme immunoassay (Biosource Europe, Nivelles, Belgium). The lower limit of the detection for IL -6 was $0.104 \mathrm{pg} / \mathrm{ml}$ and the intra- and interassay coefficients of variation were 4.71 and 6.70 respectively. This kit has no cross-reactivity with IL-2, IL-4, IL-10, IL-2R, or TNF- $\alpha$. Plasma high-sensitive CRP was determined by a highly sensitive immunonephelometry kit (Dade Behring, Marburg, Germany). In this case, both the intra- and the interassay coefficients of variation were $<5 \%$.

All microvascular complications were assessed by the same investigator, blinded to the clinical characteristics of the subjects. To evaluate CAN, the following three tests were carried out at $0800 \mathrm{~h}$, before insulin administration and after a rest period of at least $5 \mathrm{~min}(1,2)$ :

1) HRV in response to deep breathing (E/I ratio): Six maximal expirations and inspirations were performed for $1 \mathrm{~min}$, with the subjects in the supine position and during the recording of a continuous electrocardiogram. The $R-R$ intervals were registered, and the $E / I$ ratio (as a test of parasympathetic function) was calculated as the mean of the longest $R-R$ interval during expiration divided by the mean of the shortest $R-R$ interval during inspiration.

2) HRV in response to the Valsalva maneuver (Valsalva ratio): The Valsalva maneuver was carried out with the subjects in a sitting position. The subject blew into a mouthpiece and was instructed to hold a pressure of $40 \mathrm{mmHg}$ for $15 \mathrm{~s}$. During this period, the shortest $R-R$ interval was registered, and $30 \mathrm{~s}$ later, the longest $R-R$ interval was recorded. The Valsalva ratio was calculated as the longest $R-R$ interval, divided by the shortest one during and shortly after the Valsalva maneuver. 
3) Systolic blood pressure (sBP) responding to standing: After a supine rest for at least $5 \mathrm{~min}$, changes in sBP were recorded.

Each test was performed three times in each subject and the average of the three results for each test was used for data analysis. The response to each of these tests was evaluated using a portable computerized system (Cardionomic, Medimatica, Martinsicuro, Italy; (22)). Since indicated by the manufacturer, this system was able to make calculations controlling for ectopic beats. Retinopathy, nephropathy, and peripheral neuropathy were evaluated as previously described (9).

Data are presented as percentages, means \pm s.D. for variables normally distributed or medians (interquartile range) for variables not normally distributed. To improve skewness and kurtosis, variables not normally distributed were log-transformed when possible and then back-transformed to their natural units for presentation in the Tables and Fig. 2. When log transformation was not possible, appropriate nonparametric tests were used for the analyses. The potential relationships between the indexes of CAN, components of the metabolic syndrome, eGDR, and inflammation-related plasma proteins were explored using correlation tests (Pearson or Spearman's tests where appropriate). When a significant crude correlation was found, multiple linear regression models were used to adjust for potential confounding factors. To further explore such relationships, when any significant association was found between an inflammation-related plasma protein and an index of CAN, subjects were categorized into tertiles of the corresponding index of CAN (stratifying for the presence of other microvascular complications) and were compared using a one-way ANOVA and Tukey's multiple comparison post hoc test. The SPSS/PC + statistical program (v.11.5 for Windows; Chicago, IL, USA) was used for all calculations. All $P$ values were two-sided and a $P$ value $<0.05$ was considered statistically significant.

\section{Results}

One hundred and twenty subjects with type 1 diabetes were evaluated. Their clinical characteristics are shown in Table 1. Five were on treatment with low doses of converting enzyme inhibitors (enalapril or quinapril $5 \mathrm{mg}$ /day or captopril $25 \mathrm{mg} /$ day). A further five subjects were on treatment with thyroxine $\left(\mathrm{T}_{4}\right.$; dose range $50-100 \mu \mathrm{g} /$ day) and all of them had normal serum values of $\mathrm{T}_{4}$ and thyrotropin. Peripheral diabetic neuropathy was the more diagnosed microvascular complication (Table 1). No subject revealed abnormal tests for CAN although eight of them showed borderline results in at least one of these tests (Table 1), according to previous well-established cut-off points of CAN diagnosis $(23,24)$.
Table 1 Clinical characteristics of the 120 subjects with type 1 diabetes who were evaluated.

\begin{tabular}{|c|c|}
\hline & $N=120$ \\
\hline Sex (\% of women) & 50.0 \\
\hline Current age (years) & $27.4 \pm 6.6$ \\
\hline Duration of diabetes after puberty (years) & $11.9 \pm 2.8$ \\
\hline Body mass index $\left(\mathrm{kg} / \mathrm{m}^{2}\right)$ & $20.1 \pm 2.7$ \\
\hline Waist-to-hip ratio & $0.82 \pm 0.09$ \\
\hline Systolic blood pressure $(\mathrm{mmHg})$ & $113 \pm 112$ \\
\hline Diastolic blood pressure $(\mathrm{mmHg})$ & $68 \pm 9$ \\
\hline Current smokers (\%) & 58.3 \\
\hline Current alcohol intake $>20 \mathrm{~g} /$ day $(\%)$ & 24.2 \\
\hline Insulin dose (IU/day) & $51.7 \pm 16.0$ \\
\hline $\mathrm{HbA} 1 \mathrm{c}(\%)$ & $8.2(7.3-9.0)$ \\
\hline Cholesterol (mmol/l) & $4.89 \pm 0.83$ \\
\hline Triglycerides $(\mathrm{mmol} / \mathrm{l})$ & $1.46 \pm 0.37$ \\
\hline HDL-cholesterol $(\mathrm{mmol} / \mathrm{l})$ & $0.83(0 . \overline{6} 1-1.29)$ \\
\hline LDL-cholesterol (mmol/l) & $2.99 \pm 0.71$ \\
\hline Diabetic retinopathy (\%) & 15.8 \\
\hline Diabetic nephropathy (\%) & 9.2 \\
\hline Peripheral diabetic neuropathy (\%) & 27.5 \\
\hline $\begin{array}{l}\text { Subjects with at least one borderline test } \\
\text { for CAN (\%) }\end{array}$ & 6.7 \\
\hline$E / /$ ratio & $1.50 \pm 0.20$ \\
\hline Valsalva ratio & $2.00(1.61-2.34)$ \\
\hline sBP response to standing & $-1.1 \pm 8.4$ \\
\hline sTNFR1 (ng/ml) & $2.10 \pm 0.68$ \\
\hline sTNFR2 $(\mathrm{ng} / \mathrm{ml})$ & $4.31 \pm 1.19$ \\
\hline IL-6 (pg/ml) & $0.85(0 . \overline{6} 1-1.29)$ \\
\hline CRP $(\mathrm{mg} / \mathrm{dl})$ & $0.96(0.45-2.58)$ \\
\hline eGDR (mg/kg per min) & $8.00(6.88-9.01)$ \\
\hline
\end{tabular}

All values are given as percentages, means \pm s.D. or medians (interquartile range).

Table 2 shows the crude correlation coefficients between the three indexes of CAN and all the other variables, categorical or continuous, which were analyzed. Women had a higher Valsalva ratio than men. In addition, a negative correlation was found between E/I ratio and age, BMI, and diastolic BP (dBP). Valsalva ratio also correlated negatively with $\mathrm{dBP}$ and $\mathrm{HbA1c}$ levels, and a negative correlation was found between the sBP response to standing and sBP. The only significant association between an index of CAN and a plasma inflammation-related protein was found between E/I ratio and $\mathrm{IL}-6$, such being a negative correlation (Table 2, Fig. 1). Since both smoking and alcohol intake can modify measures of CAN (like E/I ratio, Valsalva ratio, and SBP responding to standing) and the evaluated inflammation-related plasma proteins (sTNFR1, sTNFR2, IL-6, and CRP), we also explored whether smoking or alcohol intake correlated with any of these measurements. In this evaluation, only a significant positive correlation between smoking and IL- 6 plasma concentrations $(r=0.257, P=0.011)$ was found. We included insulin doses in the analyses, because insulin can influence both the function of the autonomic nervous system (25) and some inflammation-related proteins (26), and because subjects with type 1 diabetes usually show high plasma concentrations of insulin due to their insulin treatment. In fact, a significant 
Table 2 Crude correlation coefficients between indexes of cardiovascular autonomic neuropathy (E/I ratio, Valsalva ratio, and systolic blood pressure (sBP) response to standing) and the other characteristics of the subjects (including components of the metabolic syndrome, estimation of the glucose disposal rate (eGDR), insulin dose, inflammation-related plasma proteins soluble tumour necrosis factor receptor 1 (sTNFR1) and 2 (sTNFR2), IL-6, and CRP).

\begin{tabular}{lccc}
\hline & Ell ratio & $\begin{array}{c}\text { Valsalva } \\
\text { ratio }\end{array}$ & $\begin{array}{c}\text { sBP } \\
\text { response to } \\
\text { standing }\end{array}$ \\
\hline Categorical variables & & & \\
Sex & -0.081 & $0.205^{\star}$ & -0.152 \\
Diabetic retinopathy & -0.082 & -0.095 & -0.100 \\
Diabetic nephropathy & -0.069 & -0.075 & 0.054 \\
Peripheral diabetic & -0.015 & 0.001 & -0.103 \\
$\quad$ neuropathy & -0.060 & -0.187 & 0.108 \\
Smoking & -0.041 & -0.037 & 0.103 \\
Alcohol intake $>$ & & & \\
20 g/day & & & \\
Continuous variables & $-0.345^{\dagger}$ & -0.103 & -0.110 \\
Age & $-0.208^{\star}$ & -0.113 & -0.164 \\
Body mass index & -0.018 & -0.080 & 0.023 \\
Waist-to-hip ratio & -0.102 & -0.113 & $-0.199^{\star}$ \\
sBP & $-0.266^{\dagger}$ & $-0.199^{\star}$ & -0.111 \\
dBP & 0.073 & -0.009 & 0.096 \\
Insulin dose & -0.077 & $-0.188^{\star}$ & 0.068 \\
HbA1c & -0.041 & -0.062 & -0.107 \\
Cholesterol & -0.013 & 0.099 & 0.015 \\
Triglycerides & -0.093 & -0.068 & -0.168 \\
HDL-cholesterol & -0.013 & -0.114 & -0.131 \\
LDL-cholesterol & 0.078 & -0.052 & 0.054 \\
STNFR1 & -0.007 & 0.077 & -0.003 \\
sTNFR2 & $-0.244^{\star}$ & -0.051 & 0.085 \\
IL-6 & 0.038 & -0.045 & 0.046 \\
CRP & 0.160 & 0.186 & -0.029 \\
eGDR & & & \\
\hline
\end{tabular}

${ }^{*} P<0.05,{ }^{\dagger} P<0.01$

relationship was found between the insulin dose and the IL-6 plasma concentrations that persisted after adjusting for age, sex, and HbA1c values $(r=0.216 ; P=0.014)$. Of note, we also found a significant positive correlation between peripheral neuropathy and the presence of at least one borderline result for the tests of CAN $(r=0.193$; $P=0.043)$. Finally, the presence of peripheral neuropathy correlated positively with the plasma concentrations of sTNFR $1(r=0.241 ; P=0.011)$ but not with any other of the evaluated inflammation-related plasma proteins.

The significant crude negative correlation between plasma concentrations of IL- 6 and $E / I$ ratio persisted after adjusting for age, sex, $\mathrm{HbAlc}$, components of the metabolic syndrome (BMI, dBP, triglycerides, and HDLcholesterol), microvascular complications (retinopathy, nephropathy, and peripheral neuropathy), and insulin dose $(r=-0.234 ; P=0.032)$. A further additional adjustment for smoking did not significantly modify this relationship $(r=-0.231 ; P=0.039)$. The exclusion of data from the few subjects taking drugs affecting $\mathrm{BP}$ or $\mathrm{T}_{4}$ did not change these results. Finally, when subjects were stratified according to tertiles of $E / I$ ratio,
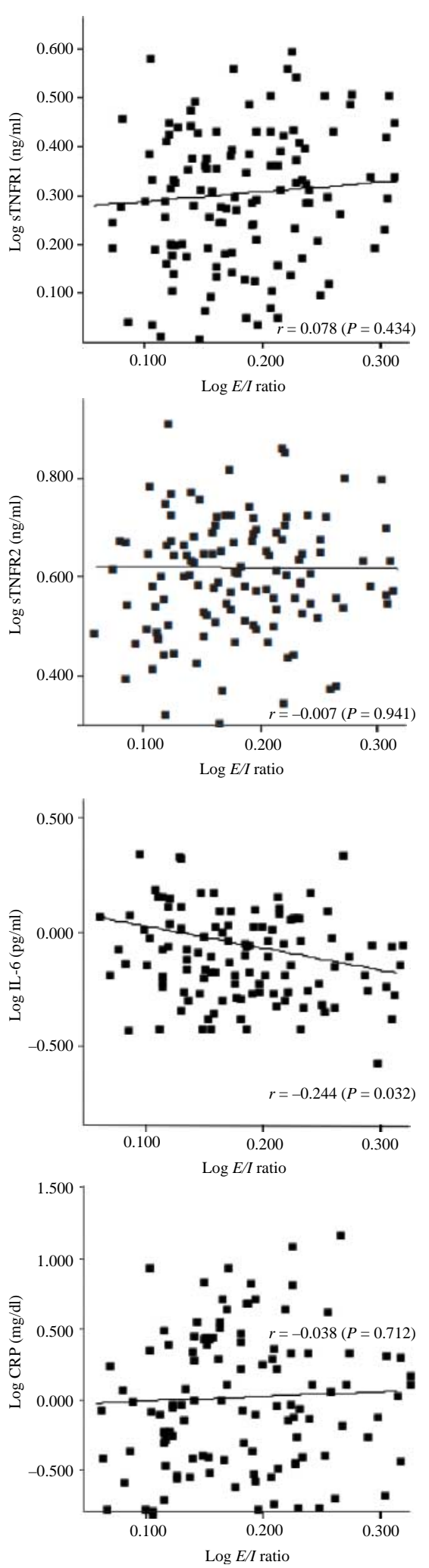

Figure 1 Scatter plots showing the crude linear correlations with their regression lines between $E / /$ ratio and plasma concentrations of sTNFR1, sTNFR2, IL-6, and CRP. All variables were log-transformed. 
significant differences were found between those in the first tertile and those in the second and third tertiles $(P$ value for $\mathrm{ANOVA}=0.031$; Tukey's post hoc tests: first versus second tertile, $P=0.042$; first versus third tertile, $P=0.047$; Fig. 2). No significant difference was found between subjects in the second and third tertiles (Tukey's post hoc test: $P=0.242$; Fig. 2). This analysis was also performed stratifying subjects into two groups, with or without other microvascular complications. Analysis in subjects with microvascular complications did not find differences between the three tertiles $(P$ value for ANOVA $=0.179$ ). Analysis in subjects without other microvascular complications resembled those in the whole group ( $P$ value for ANOVA $=0.026$; Tukey's post hoc tests: first versus second tertile, $P=0.041$; first versus third tertile, $P=0.046$; second versus third tertile, $P=0.185)$.

\section{Discussion}

This is the first study linking low-grade systemic inflammation and indexes of CAN in type 1 diabetes. Its main finding is that after 14 years of evolution of type 1 diabetes, plasma concentrations of IL- 6 increase as $E / I$ ratio decreases. This relationship is moderate but persists even after adjusting for potential confounding factors such as age, sex, and glycemic control. Further adjustments for components of the metabolic syndrome, other microvascular complications, insulin doses, or

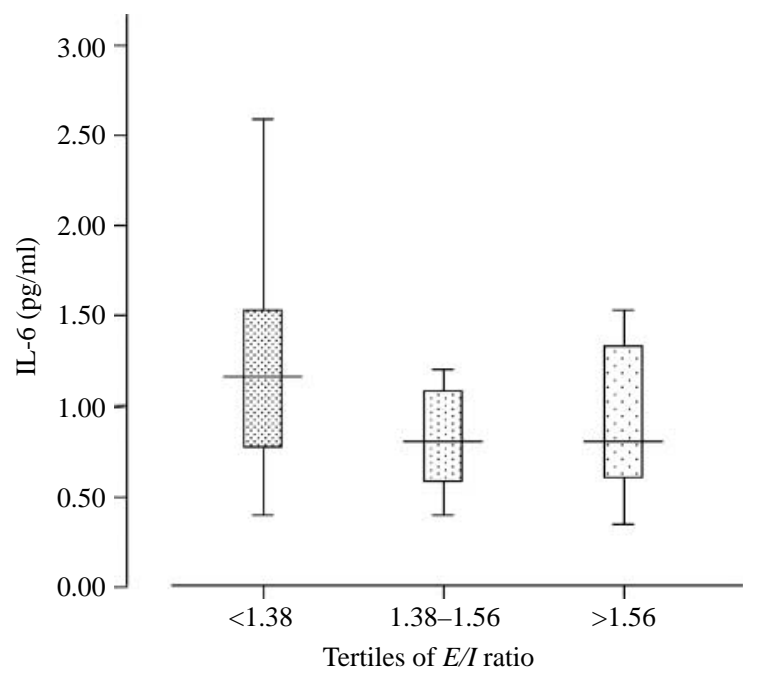

Figure 2 Serum concentrations of IL-6 according to the tertiles of E/I ratio. The lower and the upper boundaries of the boxes indicate the interquartile range, and the lines within the boxes mark the median. Error bars above and below the box include the 10th and 90th percentiles respectively. Subjects in the first tertile of $E / I$ ratio had higher concentrations of IL-6 than subjects in the second and third tertiles ( $P$ value for ANOVA $=0.031$; Tukey's post hoc tests: $P=$ 0.042 and $P=0.047$ respectively). No differences were found between subjects in the second and third tertiles (Tukey's post hoc test: $P=0.242$ ). smoking do not substantially change this relationship. Since a decrease in E/I ratio is considered as an early sign of CAN, this finding suggests that subjects with early signs of CAN have an increase in plasma concentrations of IL-6. This finding might be of importance regarding the pathogenesis of CAN and/or in its clinical consequences.

The negative correlation between $E / I$ ratio and plasma concentrations of IL- 6 is supported by previous cross-sectional studies which evaluated the relationship between HRV and low-grade systemic inflammation. These studies have been carried out in healthy subjects $(18,19)$ and in patients with the metabolic syndrome (27), coronary heart disease (28), and heart failure (29). In these studies, blood leucocyte counts, IL-6, CRP, and TNF- $\alpha$ have been used as markers of low-grade systemic inflammation. Although none of them have simultaneously evaluated IL-6, CRP, and the TNF- $\alpha$ system, IL- 6 was the inflammation-related protein more consistently correlated with lower HRV (27-29). In addition, the level of negative correlation between IL-6 and HRV found in these studies was of similar magnitude to the one in the current study $(27,28)$. To our knowledge, only one previous study has tested our hypothesis in subjects with diabetes mellitus. This study was carried out in a small group of 17 subjects with type 2 diabetes and 18 controls, and found that a depression in reflex vagal function was associated with an increase in plasma concentrations of CRP (30). However, this relationship disappeared in the multivariate analyses and a sizeable proportion of the subjects were on pharmacological treatment for hypertension and dyslipemia.

The negative correlation between E/I ratio and IL-6 may be explained in several ways. First, CAN has been associated with several cardiovascular risk factors included in the metabolic syndrome, both in population-based studies (31) and in type 1 diabetes $(5,17)$. Since insulin resistance correlates with both these cardiovascular risk factors and IL-6 (12), it could be debated that the negative correlation between $E / I$ ratio and plasma concentrations of IL- 6 could be explained by an increase in insulin resistance in subjects with a lower E/I ratio. However, the analyses of our data dispute this possibility as the only mechanism linking E/I ratio and IL-6, because this relationship remained substantially unchanged even after adjusting for factors determining insulin resistance in type 1 diabetes. An alternative explanation for the negative correlation between $E / I$ ratio and IL- 6 comes from the interactions between the autonomic and the immune systems. This interaction is biologically plausible, since the autonomic nervous system can control the release of IL-6 (32) and, in turn, IL-6 can influence the autonomic nervous system. A decrease in E/I ratio is considered to be a sign of parasympathetic dysfunction (1) and it is known that acetylcholine, the main parasympathetic neurotransmitter, acting on specific receptors on macrophages 
significantly diminishes the release of pro-inflammatory cytokines, such as TNF- $\alpha$ or IL-6 (33). In addition, the sympathetic nervous system acting through the $\beta$-adrenergic receptor increases IL-6 production (34). So, it is tempting to speculate that the increase in IL-6 as E/I ratio decreases in the current study could be a reflection of the imbalance between parasympathetic and sympathetic function. However, the opposite may also be true: IL- 6 can influence the autonomic nervous system. In this regard, it has been demonstrated that IL- 6 may stimulate the sympathetic nervous system (35). In addition, some evidence suggests that IL- 6 may play a pathogenic role in diabetic neuropathy. Although there are no data available on autonomic nerves, in sural nerve biopsies in subjects with diabetic neuropathy it has been shown that IL-6 co-localizes in the microvasculature with advanced glycation end products, the receptor of these products, and the activation of the NFкB pathway (36). Other studies suggested that IL- 6 may play a major role in the process of nerve regeneration $(37,38)$ or, conversely, that IL-6 can promote nerve degeneration (39).

Regardless of the mechanisms implicated in the increase of IL- 6 in subjects with lower E/I ratio, these mechanisms would even operate with slight disturbances in the autonomic nervous system function, because most of the subjects in our study had normal tests for CAN. In addition, this increase in IL-6 may be of importance regarding the clinical consequences of CAN. Mortality is higher in subjects with CAN due to myocardial ischemia, among other conditions $(1,40)$. Consistent with this notion, IL-6 is involved in the atherosclerotic process (41), and is a predictor of cardiovascular events (42). In addition, subjects with CAN also seem to have an increased risk of heart failure (1). Accordingly, IL-6 may induce contractile myocardial dysfunction (43) and may be implicated in the pathogenesis of heart failure (44).

Plasma concentrations of IL- 6 can be decreased through lifestyle interventions $(45,46)$ or using pharmacological agents (47). So, one additional potential interest of our study is that these therapeutic strategies could be used either to prevent CAN or the consequences of an increase in IL- 6 associated with the development of CAN.

Another interesting point raised in the present study is that we found an inverse relationship between $E / I$ ratio and IL-6, but not between E/I ratio and sTNFR1, sTNFR2, or CRP. As stated above, IL- 6 has been the inflammation-related protein most consistently associated with lower HRV, and these differences may reflect real pathophysiological differences in how these proteins relate to the activity of the autonomic nervous system. Alternatively, if we assume that this relationship is explained by the interactions between the autonomic and the immune systems, it should be noted that, for example, there is some evidence that the autonomic nervous system could modulate IL- 6 and TNF- $\alpha$ differently (48). Clearly, further studies are needed to address this issue.

The present study has certain limitations. First, two matters of concern are the selection bias and sample size. It could be debated that the frequency of CAN (and that of the other microvascular complications) in the subjects of the current study was lower than expected, indicating some degree of selection bias. In addition, the sample size of the present study was relatively small. However, under these circumstances, any possible relationship between indexes of CAN, insulin resistance, and inflammation-related proteins would have been underestimated. These circumstances could justify the moderate relationship found between IL- 6 and E/I ratio, although, as stated before, this correlation was in the same range as in previous studies in other populations $(27,28)$. In addition, they could also help to explain why we did not find any significant relationship between HRV and HbA1c levels, a finding reported in other studies $(6,49,50)$ or between HRV and insulin resistance, a finding reported in other populations, but not previously assessed in subjects with type 1 diabetes (31). In this regard, we also acknowledge the limitations of the formula of Williams et al. (20) to evaluate the insulin resistance in subjects with type 1 diabetes. This formula has been used in this context in other previously published studies, but it should be borne in mind that it is based exclusively on clinical factors. Another limitation of the current study has to do with its cross-sectional design. Because of this, it was not possible to determine whether reduced $E / I$ ratio precedes elevated IL-6 levels or is a consequence of it. In addition, we cannot exclude that this relationship could be explained by the existence of one or more unmeasured factors associated with both the decrease of $E / I$ ratio and the increase in IL- 6 . In spite of all these limitations, our study provides a novel view on how the autonomous nervous system and low-grade systemic inflammation could relate to each other in type 1 diabetes.

In conclusion, the present results suggest a link between IL- 6 and early alterations of CAN in type 1 diabetes, regardless of insulin resistance or other known confounding factors. At present, it is not clear which mechanisms can explain this association, but IL-6 might represent a new mechanism involved in the pathogenesis of CAN and/or in its clinical consequences.

\section{Acknowledgements}

We thank the Fondo de Investigaciones Sanitarias (RG03/212, RC03/08) for financial support. We are indebted to Dr Conxa Castell (Consell Assesor sobre la Diabetis a Catalunya), Neil Hossack, and the Board of the Associació Catalana de Diabetis for their assistance in the development of this study. The authors declare 
that there is no conflict of interest that would prejudice the impartiality of this scientific work.

\section{References}

1 Vinik AI, Maser RE, Mitchell BD \& Freeman R. Diabetic autonomic neuropathy. Diabetes Care 200326 1553-1579.

2 Proceedings of a consensus development conference on standardized measures in diabetic neuropathy. Summary and recommendations. Diabetes Care 199215 1104-1107.

3 Assessment: Clinical autonomic testing report of the Therapeutics and Technology Assessment Subcommittee of the American Academy of Neurology. Neurology 199646 873-880.

4 The effect of intensive diabetes therapy on measures of autonomic nervous system function in the Diabetes Control and Complications Trial (DCCT). Diabetologia 199841 416-423.

5 Kempler P, Tesfaye S, Chaturvedi N, Stevens LK, Webb DJ, Eaton S, Kerényi Z, Tamás G, Ward JD \& Fuller JH. Autonomic neuropathy is associated with increased cardiovascular risk factors: the EURODIAB IDDM Complications Study. Diabetic Medicine 2002 19 900-909.

6 Colhoun HM, Francis DP, Rubens MB, Underwood SR \& Fuller JH. The association of heart-rate variability with cardiovascular risk factors and coronary artery calcification: a study in type 1 diabetic patients and the general population. Diabetes Care 200124 1108-1114.

7 Orchard TJ, Chang YF, Ferrell RE, Petro N \& Ellis DE. Nephropathy in type 1 diabetes: a manifestation of insulin resistance and multiple genetic susceptibilities? Further evidence from the Pittsburgh Epidemiology of Diabetes Complication Study. Kidney International 200262 963-970.

8 Schram MT, Chaturvedi N, Schalkwijk CG, Fuller JH \& Stehouwer CD. Markers of inflammation are cross-sectionally associated with microvascular complications and cardiovascular disease in type 1 diabetes-the EURODIAB Prospective Complications Study. Diabetologia 200548 370-378.

9 Gonzalez-Clemente JM, Mauricio D, Richart C, Broch M, Caixas A, Megia A, Gimenez-Palop O, Simon I, Martinez-Riquelme A, Gimenez-Perez G \& Vendrell J. Diabetic neuropathy is associated with activation of the TNF- $\alpha$ system in subjects with type 1 diabetes mellitus. Clinical Endocrinology 200563 525-529.

10 Orchard TJ, Olson JC, Erbey JR, Williams K, Forrest KY, Smithline Kinder L, Ellis D \& Becker DJ. Insulin resistance-related factors, but not glycemia, predict coronary artery disease in type 1 diabetes: 10-year follow-up data from the Pittsburgh Epidemiology of Diabetes Complications Study. Diabetes Care 200326 1374-1379.

11 Schram MT, Chaturvedi N, Schalkwijk C, Giorgino F, Ebeling P, Fuller JH \& Stehouwer CD. Vascular risk factors and markers of endothelial function as determinants of inflammatory markers in type 1 diabetes: the EURODIAB Prospective Complications Study. Diabetes Care 200326 2165-2173.

12 Fernandez-Real JM \& Ricart W. Insulin resistance and chronic cardiovascular inflammatory syndrome. Endocrine Reviews 2003 24 278-301.

13 Zoppini G, Faccini G, Muggeo M, Zenari L, Falezza G \& Targher G. Elevated plasma levels of soluble receptors of TNF- $\alpha$ and their association with smoking and microvascular complications in young adults with type 1 diabetes. Journal of Clinical Endocrinology and Metabolism 200186 3805-3808.

14 Foss CH, Vestbo E, Froland A, Gjessing HJ, Mogensen CE \& Damsgaard EM. Autonomic neuropathy in nondiabetic offspring of type 2 diabetic subjects is associated with urinary albumin excretion rate and 24-h ambulatory blood pressure: the Fredericia Study. Diabetes 200150 630-636.

15 Takayama S, Sakura H, Katsumori K, Wasada T \& Iwamoto Y. A possible involvement of parasympathetic neuropathy on insulin resistance in patients with type 2 diabetes. Diabetes Care 200124 968-969.
16 Huikuri HV, Ylitalo A, Pikkujamsa SM, Ikaheimo MJ, Airaksinen KE, Rantala AO, Lilja M \& Kesaniemi YA. Heart rate variability in systemic hypertension. American Journal of Cardiology 199677 1073-1077.

17 Maser RE, Pfeifer MA, Dorman JS, Kuller LH, Becker DJ \& Orchard TJ. Diabetic autonomic neuropathy and cardiovascular risk. Pittsburgh Epidemiology of Diabetes Complications Study III. Archives of Internal Medicine 1990150 1218-1222.

18 Jensen-Urstad M, Jensen-Urstad K, Ericson M \& Johansson J. Heart rate variability is related to leucocyte count in men and to blood lipoproteins in women in a healthy population of 35-year-old subjects. Journal of Internal Medicine 1998243 33-40.

19 Sajadieh A, Nielsen OW, Rasmussen V, Hein HO, Abedini S \& Hansen JF. Increased heart rate and reduced heart-rate variability are associated with subclinical inflammation in middle-aged and elderly subjects with no apparent heart disease. European Heart Journal 200425 363-370.

20 Williams KV, Erbey JR, Becker D, Arslanian S \& Orchard TJ. Can clinical factors estimate insulin resistance in type 1 diabetes? Diabetes $200049626-632$.

21 Goday A, Castell C, Tresserras R, Canela J, Taberner JL \& Lloveras G. Incidence of type 1 (insulin-dependent) diabetes mellitus in Catalonia, Spain. The Catalan Epidemiology Diabetes Study Group. Diabetologia 199235 267-271.

22 Vespasiani G, Bruni M, Meloncelli I, Clementi L, Amoretti R, Branca S, Carinci F, Lostia S, Nicolucci A, Romagnoli F, Verga S \& Benedetti MM. Validation of a computerised measurement system for guided routine evaluation of cardiovascular autonomic neuropathy. Computer Methods and Programs in Biomedicine 1996 $51211-216$.

23 Ewing DJ, Martyn CN, Young RJ \& Clarke BF. The value of cardiovascular autonomic function tests: 10 years experience in diabetes. Diabetes Care 19858 491-498.

24 Lluch I, Hernandez A, Real JT, Morillas C, Tenes S, Sanchez C \& Ascaso JF. Cardiovascular autonomic neuropathy in type 1 diabetic patients with and without peripheral neuropathy. Diabetes Research and Clinical Practice 199842 35-40.

25 Bergholm R, Westerbacka J, Vehkavaara S, Seppälä-Lindroos A, Goto T \& Yki-Järvinen H. Insulin sensitivity regulates autonomic control of heart rate variation independent of body weight in normal subjects. Journal of Clinical Endocrinology and Metabolism 200186 1403-1409.

26 Krogh-Madsen R, Plomgaard P, Keller P, Keller C \& Pedersen BK. Insulin stimulates interleukin- 6 and tumor necrosis factor-alpha gene expression in human subcutaneous adipose tissue. American Journal of Physiology. Endocrinology and Metabolism 2004286 E234-E238.

27 Brunner EJ, Hemingway H, Walker BR, Page M, Clarke P, Juneja M, Shipley MJ, Kumari M, Andrew R, Seckl JR, Papadopoulos A, Checkley S, Rumley A, Lowe GD, Stansfeld SA \& Marmot MG. Adrenocortical, autonomic, and inflammatory causes of the metabolic syndrome: nested case-control study. Circulation 2002 $1062659-2665$.

28 Janszky I, Ericson M, Lekander M, Blom M, Buhlin K, Georgiades A \& Ahnve S. Inflammatory markers and heart rate variability in women with coronary heart disease. Journal of Internal Medicine $2004256421-428$.

29 Aronson D, Mittleman MA \& Burger AJ. Interleukin-6 levels are inversely correlated with heart rate variability in patients with decompensated heart failure. Journal of Cardiovascular Electrophysiology 200112 294-300.

30 Anan F, Takahashi N, Nakagawa M, Ooie T, Saikawa T \& Yoshimatsu H. High-sensitivity C-reactive protein is associated with insulin resistance and cardiovascular autonomic dysfunction in type 2 diabetic patients. Metabolism 200554 552-558.

31 Liao D, Sloan RP, Cascio WE, Folsom AR, Liese AD, Evans GW, Cai J \& Sharrett AR. Multiple metabolic syndrome is associated with lower heart rate variability. The Atherosclerosis Risk in Communities Study. Diabetes Care 199821 2116-2122.

32 Tracey KJ. The inflammatory reflex. Nature 2002 420 853-859. 
33 Borovikova LV, Ivanova S, Zhang M, Yang H, Botchkina GI, Watkins LR, Wang H, Abumrad N, Eaton JW \& Tracey KJ. Vagus nerve stimulation attenuates the systemic inflammatory response to endotoxin. Nature $2000 \mathbf{4 0 5} 458-462$.

34 Mohamed-Ali V, Flower L, Sethi J, Hotamisligil G, Gray R, Humphries SE, York DA \& Pinkney J. $\beta$-Adrenergic regulation of IL-6 release from adipose tissue: in vivo and in vitro studies. Journal of Clinical Endocrinology and Metabolism 2001865864 5869.

35 Besedovsky HO \& del Rey A. Immune-neuro-endocrine interactions: facts and hypotheses. Endocrine Reviews $1996 \mathbf{1 7}$ 64-102.

36 Bierhaus A, Haslbeck KM, Humpert PM, Liliensiek B, Dehmer T, Morcos M, Sayed AA, Andrassy M, Schiekofer S, Schneider JG, Schulz JB, Heuss D, Neundorfer B, Dierl S, Huber J, Tritschler H, Schmidt AM, Schwaninger M, Haering HU, Schleicher E, Kasper M, Stern DM, Arnold B \& Nawroth PP. Loss of pain perception in diabetes is dependent on a receptor of the immunoglobulin superfamily. Journal of Clinical Investigation $20041141741-1751$.

37 Cafferty WB, Gardiner NJ, Das P, Qiu J, McMahon SB \& Thompson SW. Conditioning injury-induced spinal axon regeneration fails in interleukin-6 knock-out mice. Journal of Neuroscience 200424 4432-4443.

38 Skundric DS, Dai R \& Mataverde P. IL-6 modulates hyperglycemiainduced changes of $\mathrm{Na}+$ channel Beta-3 subunit expression by Schwann cells. Annals of the New York Academy of Sciences 2003 1005 233-236.

39 Kaplin AI, Deshpande DM, Scott E, Krishnan C, Carmen JS, Shats I, Martinez T, Drummond J, Dike S, Pletnikov M, Keswani SC, Moran TH, Pardo CA, Calabresi PA \& Kerr DA. IL-6 induces regionally selective spinal cord injury in patients with the neuroinflammatory disorder transverse myelitis. Journal of Clinical Investigation $2005 \mathbf{1 1 5} 2731-2741$.

40 Wackers FJ, Young LH, Inzucchi SE, Chyun DA, Davey JA, Barrett EJ, Taillefer R, Wittlin SD, Heller GV, Filipchuk N, Engel S, Ratner RE \& Iskandrian AE. Detection of silent myocardial ischemia in asymptomatic diabetic subjects: the DIAD study. Diabetes Care 200427 1954-1961.

41 Yudkin JS, Kumari M, Humphries SE \& Mohamed-Ali V. Inflammation, obesity, stress and coronary heart disease: is interleukin-6 the link? Atherosclerosis 2000148 209-214.
42 Ridker PM, Rifai N, Stampfer MJ \& Hennekens CH. Plasma concentration of interleukin-6 and the risk of future myocardial infarction among apparently healthy men. Circulation $2000 \mathbf{1 0 1}$ 1767-1772.

43 Prabhu SD. Cytokine-induced modulation of cardiac function. Circulation Research 200495 1140-1153.

44 Marfella R, Esposito K, Siniscalchi M, Cacciapuoti F, Giugliano F, Labriola D, Ciotola M, Di Palo C, Misso L \& Giugliano D. Effect of weight loss on cardiac synchronization and proinflammatory cytokines in premenopausal obese women. Diabetes Care 200427 47-52.

45 Esposito K, Pontillo A, Di Palo C, Giugliano G, Masella M, Marfella R \& Giugliano D. Effect of weight loss and lifestyle changes on vascular inflammatory markers in obese women: a randomized trial. JAMA 2003289 1799-1804.

46 Dekker MJ, Lee S, Hudson R, Kilpatrick K, Graham TE, Ross R \& Robinson LE. An exercise intervention without weight loss decreases circulating interleukin-6 in lean and obese men with and without type 2 diabetes mellitus. Metabolism 200756 332-338.

47 Samaha FF, Szapary PO, Iqbal N, Williams MM, Bloedon LT, Kochar A, Wolfe ML \& Rader DJ. Effects of rosiglitazone on lipids, adipokines, and inflammatory markers in nondiabetic patients with low high-density lipoprotein cholesterol and metabolic syndrome. Arteriosclerosis, Thrombosis, and Vascular Biology 2006 26 624-630.

48 Goebel MU, Mills PJ, Irwin MR \& Ziegler MG. Interleukin-6 and tumor necrosis factor-alpha production after acute psychological stress, exercise, and infused isoproterenol: differential effects and pathways. Psychosomatic Medicine 2000 62 591-598.

49 Valensi P, Huard JP, Giroux C \& Attali JR. Factors involved in cardiac autonomic neuropathy in diabetic patients. Journal of Diabetes and its Complications 199711 180-187.

50 Gottsater A, Kangro M \& Sundkvist G. Early parasympathetic neuropathy associated with elevated fasting plasma C-peptide concentrations and late parasympathetic neuropathy with hyperglycaemia and other microvascular complications. Diabetic Medicine 200421 1304-1309.

Received 12 February 2007

Accepted 3 May 2007 